5-1-2015

\title{
Estimation for the Parameters of the Exponentiated Exponential Distribution Using a Median Ranked Set Sampling
}

Monjed H. Samuh

Palestine Polytechnic University, monjedsamuh@ppu.edu

Areen Qtait

Palestine Polytechnic University, areen_cutegirl@hotmail.com

Follow this and additional works at: http://digitalcommons.wayne.edu/jmasm

Part of the Applied Statistics Commons, Social and Behavioral Sciences Commons, and the Statistical Theory Commons

\section{Recommended Citation}

Samuh, Monjed H. and Qtait, Areen (2015) "Estimation for the Parameters of the Exponentiated Exponential Distribution Using a Median Ranked Set Sampling," Journal of Modern Applied Statistical Methods: Vol. 14 : Iss. 1 , Article 19. DOI: $10.22237 /$ jmasm/1430453880

Available at: http://digitalcommons.wayne.edu/jmasm/vol14/iss1/19 


\section{Estimation for the Parameters of the Exponentiated Exponential Distribution Using a Median Ranked Set Sampling}

\author{
Monjed H. Samuh \\ Palestine Polytechnic University \\ Palestinian Territories
}

\author{
Areen Qtait \\ Palestine Polytechnic University \\ Palestinian Territories
}

The method of maximum likelihood estimation based on Median Ranked Set Sampling (MRSS) was used to estimate the shape and scale parameters of the Exponentiated Exponential Distribution (EED). They were compared with the conventional estimators. The relative efficiency was used for comparison. The amount of information (in Fisher's sense) available from the MRSS about the parameters of the EED were be evaluated. Confidence intervals for the parameters were constructed using MRSS.

Keywords: $\quad$ Exponentiated exponential distribution; Fisher information; Maximum likelihood estimation; Median ranked set sampling; Ranked set sampling

\section{Introduction}

One of the most common approaches of data collection is that of a simple random sample (SRS). Other more structured sampling designs, such as stratified sampling or probability sampling, are also available to help make sure that the obtained data collection provides a good representation of the population of interest. Any such additional structure of this type revolves around how the sample data themselves should be collected in order to provide an informative image of the larger population. With any of these approaches, once the sample items have been chosen, the desired measurements are collected from each of the selected items.

Many efforts are made to develop statistical techniques for data collection that generally leads to more representative samples (samples whose characteristics accurately reflect those of the underlying population). To this end, ranked set sampling and some of its variations were developed.

Dr. Samuh is Assistant Professor of Statistics in the College of Applied Sciences. Email himat monjedsamuh@ppu.edu. 


\section{ESTIMATION FOR PARAMETERS OF THE EED USING MRSS}

In this section, the ranked set sampling (RSS) and median ranked set sampling (MRSS) are presented. The exponentiated exponential distribution (EED) and its properties are also discussed.

\section{Ranked set sampling}

RSS was proposed by McIntyre (1952) to estimate a pasture yield in Australia. This method was not used for a long time, but in the last 30 years a lot of research work was done using this method, which has become very important in different aspects.

SRS and RSS are both independent, but they differ in several ways, like:

1. RSS is more efficient than SRS with the same number of measured elements.

2. Development of RSS procedure is more difficult than that of SRS.

3. In SRS, just $m$ elements are needed but in RSS $m$ elements are chosen out of $m^{2}$ to achieve the desired sample.

Also stratified random sampling and RSS are different in some things like:

1. In stratified sampling we limited with no more six strata but in RSS we are not restricted ourselves with the number of sets.

2. In both of them SRS is used but in RSS ordering the elements in each set is needed before selecting the sample.

RSS as a method used basically for infinite population where the set of sampling units drawn from a population can ranked in a cheap way which is not costly and/or time consuming. The steps of choosing RSS are as follows:

1. Randomly select $m$ sets each of size $m$ elements from the population under study.

2. The elements for each set in Step (1) are ranked visually or by any negligible cost method that does not require actual measurements.

3. Select and quantify the $i^{\text {th }}$ minimum from the $i^{\text {th }}$ set, $i=1,2, \ldots, m$ to get a new set of size $m$, which is called the ranked set sample.

4. Repeat Steps (1) - (3) $h$ times (cycles) until obtaining a sample of size $n=m h$. 


\section{SAMUH \& QTAIT}

Figure 1 illustrates the procedure of RSS in terms of matrices. Let $Y_{i}=\left\{X_{(i i)} ; i=1, \ldots, m\right\}$; that is, the obtained RSS, $\left\{X_{(11)}, X_{(22)}, \ldots, X_{(m m)}\right\}$, is denoted by $\left\{Y_{1}, Y_{2}, \ldots, Y_{m}\right\}$. If the process is repeated $h$ cycles, then the RSS can be represented as a matrix of size $n=h m$ as it is shown in Step 4 of Figure 1.

$$
\text { Step 1: }\left[\begin{array}{cccc}
X_{11} & X_{12} & \cdots & X_{1 m} \\
X_{21} & X_{22} & \cdots & X_{2 m} \\
\vdots & \vdots & \ddots & \vdots \\
X_{m 1} & X_{m 2} & \cdots & X_{m m}
\end{array}\right]
$$

Step 3: $\left\{X_{(11)}, X_{(22)}, \ldots, X_{(m m)}\right\}$
Step 2:

$$
\left[\begin{array}{cccc}
X_{(11)} & X_{(12)} & \cdots & X_{(1 m)} \\
X_{(21)} & X_{(22)} & \cdots & X_{(2 m)} \\
\vdots & \vdots & \ddots & \vdots \\
X_{(m 1)} & X_{(m 2)} & \cdots & X_{(m m)}
\end{array}\right]
$$

Step 4: $\left[\begin{array}{cccc}Y_{11} & Y_{12} & \cdots & Y_{1 m} \\ Y_{21} & Y_{22} & \cdots & Y_{2 m} \\ \vdots & \vdots & \ddots & \vdots \\ X_{h 1} & X_{h 2} & \cdots & X_{h m}\end{array}\right]$

Figure 1. Ranked set sampling procedure

RSS as a method is applicable where ranking and sampling units is much cheaper than the measurement of the variable of interest. In particular RSS can be used in the following situation:

1. Ranking units in a set can be done easily by judgment in the variable of interest through visual inspection or with the help of certain auxiliary means.

2. If there is a concomitant variable can be obtained easily (concomitant is a variable which is not of major concern but are correlated with the variable of interest).

\section{Median ranked set sampling}

MRSS was suggested by Muttlak (1997) as a method to estimate the population mean instead of RSS to reduce the errors, and increase the efficiency over RSS and SRS. It is described by the following steps: 


\section{ESTIMATION FOR PARAMETERS OF THE EED USING MRSS}

1. Randomly select $m^{2}$ sample units from the target population.

2. Allocate the $m^{2}$ units into $m$ sets each of size $m$, and rank the units within each set.

3. From each set in Step (2), if the sample size $m$ is odd select from each set the $\left(\frac{m+1}{2}\right)^{\text {th }}$ smallest rank unit i.e the median of each set. While if the sample size $m$ is even select from the first $\frac{m}{2}$ sets the $\left(\frac{m}{2}\right)^{t h}$ smallest rank unit and from the second $\frac{m}{2}$ sets take the $\left(\frac{m+2}{2}\right)^{\text {th }}$ smallest rank unit. This step yields $m$ sample elements which is the median RSS.

4. Repeat Steps (1) - (3) $h$ times (cycles) until obtaining a sample of size $n=m h$.

Figure 2 illustrates the procedure of MRSS when $m=4$ in terms of matrices.

Let us denote the MRSS $\left\{X_{(12)}, X_{(22)}, X_{(33)}, X_{(43)}\right\}$ by $\left\{Y_{1}, Y_{2}, Y_{3}, Y_{4}\right\}$. If the process is repeated $h$ cycles, then the RSS can be represented as a matrix of size $n=4 h$ as it is shown in Step 4 of Figure 2.

Step 1:

$$
\left[\begin{array}{llll}
X_{11} & X_{12} & X_{13} & X_{14} \\
X_{21} & X_{22} & X_{23} & X_{24} \\
X_{31} & X_{32} & X_{33} & X_{34} \\
X_{41} & X_{42} & X_{43} & X_{44}
\end{array}\right]
$$

Step 3: $\left\{X_{(12)}, X_{(22)}, X_{(33)}, X_{(44)}\right\}$
Step 2: $\left[\begin{array}{llll}X_{(11)} & X_{(12)} & X_{(13)} & X_{(14)} \\ X_{(21)} & X_{(22)} & X_{(23)} & X_{(24)} \\ X_{(31)} & X_{(32)} & X_{(33)} & X_{(34)} \\ X_{(41)} & X_{(42)} & X_{(43)} & X_{(44)}\end{array}\right]$

$$
\text { Step 4: }\left[\begin{array}{cccc}
Y_{11} & Y_{12} & Y_{13} & Y_{14} \\
Y_{21} & Y_{22} & Y_{23} & Y_{24} \\
\vdots & \vdots & \vdots & \vdots \\
Y_{h 1} & Y_{h 2} & Y_{h 3} & Y_{h 4}
\end{array}\right]
$$

Figure 2. Median ranked set sampling procedure 


\section{The exponentiated exponential distribution}

The exponentiated exponential distribution (EED) introduced by Gupta and Kundu (1999) as a generalization of the exponential distribution. It is of great interest and is popularly used in analyzing lifetime or survival data. Consider the random variable $X$ that is exponentiated exponential-distributed with scale parameter $\lambda>0$ and shape parameter $\alpha>0$. The probability density function of $X$ is given by

$$
f(x ; \alpha, \lambda)=\alpha \lambda e^{-\lambda x}\left(1-e^{-\lambda x}\right)^{\alpha-1} ; x>0 .
$$

The corresponding cumulative distribution function is given by

$$
F(x ; \alpha, \lambda)=\left(1-e^{-\lambda x}\right)^{\alpha}
$$

It is clear that the EED is simply the $\alpha^{\text {th }}$ power of the exponential cumulative distribution. So, the case where $\alpha=1$ is called the exponential distribution. The mean, variance, skewness, kurtosis and the pdf's curves of the EED for different values of the scale and shape parameters are shown in Table 1.

The properties of the EED have been studied by many authors, see for example Gupta and Kundu (2001), Nadarajah (2011), Ghitany et al. (2013), and Ristić and Nadarajah (2014).

\section{Literature Review}

Stokes (1976) used RSS for estimating the parameters in a location-scale family of distributions. The RSS estimators of the location and scale parameters are shown to be more efficient than the SRS estimators. She also used RSS to estimate the correlation coefficient of a bivariate normal distribution.

Lam et al. (1994) used RSS for estimating two-parameter exponential distribution.

$$
f(y)=\frac{1}{\sigma} \exp \left\{\frac{-(y-\theta)}{\sigma}\right\}
$$




\section{ESTIMATION FOR PARAMETERS OF THE EED USING MRSS}

Table 1. The mean, variance, skewness, kurtosis and the pdf's curves of the EED for different values of $\alpha$ and $\lambda$.

$(\alpha, \lambda)$

Properties of the EED

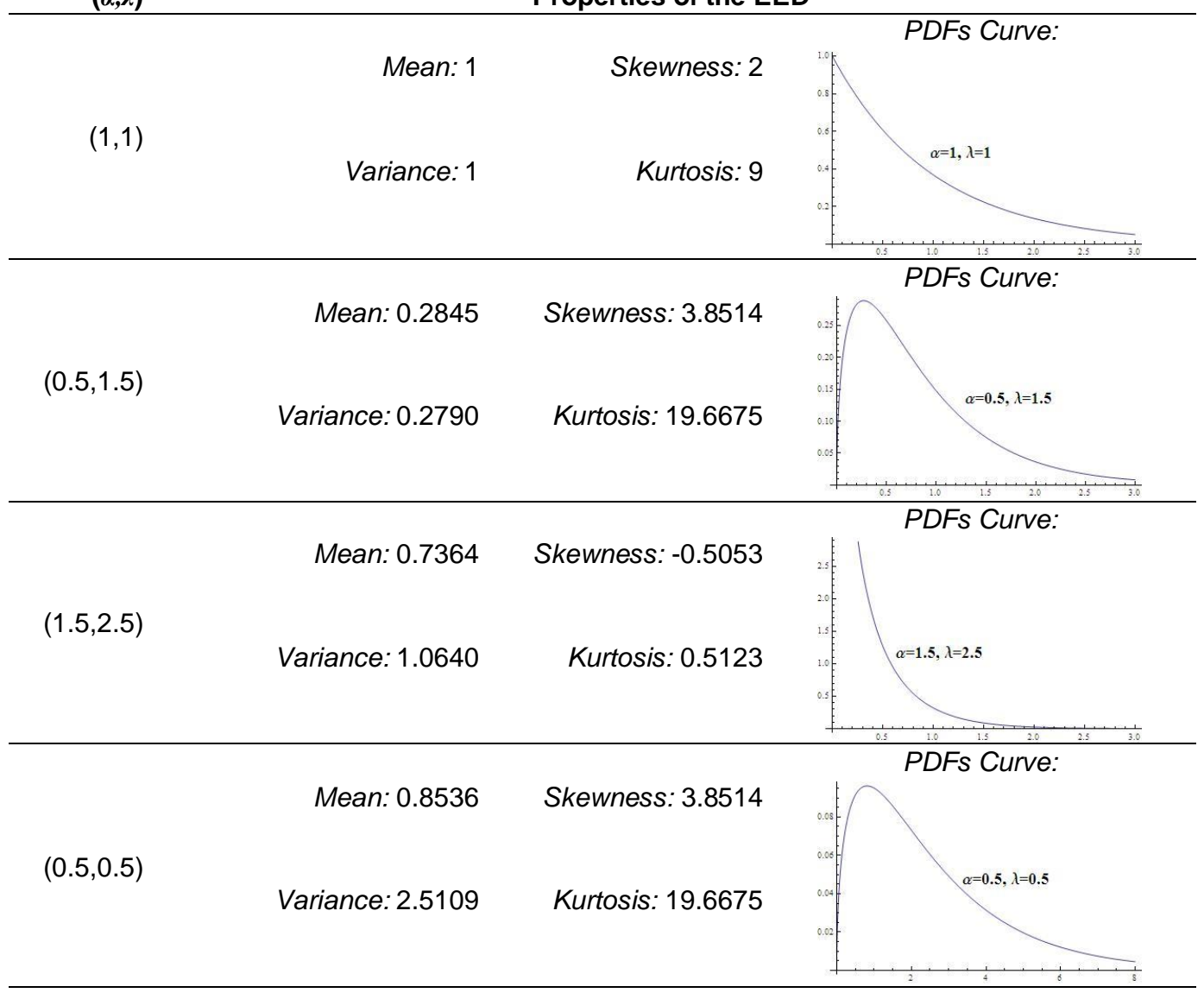

An unbiased estimators of $\theta$ and $\sigma$ based on RSS with their variances are derived. They made a comparison between these estimators with their counterpart in SRS.

Stokes (1995) considered the location-scale distribution, $F\left(\frac{x-\mu}{\sigma}\right)$, and estimated $\mu$ and $\sigma$ using the methods of maximum likelihood estimation and best linear unbiased estimation within the framework of RSS. Sinha et al. (1996) used RSS to estimate the parameters of the normal and exponential distributions. Their work assumed partial knowledge of the underlying distribution without any knowledge of the parameters. For each parameter, they proposed best linear unbiased estimators for full and partial RSS. 


\section{SAMUH \& QTAIT}

Samawi and Al-Sagheer (2001) studied the use of Extreme RSS (ERSS) and MRSS for distribution function estimation. For a random variable $X$, it is shown that the distribution function estimator when using ERSS and MRSS are more efficient than when using SRS and RSS for some values of a given $x$.

Abu-Dayyeh and Sawi (2009) considered the maximum likelihood estimator and the likelihood ratio test for making inference about the scale parameter of the exponential distribution in case of moving extreme ranked set sampling (MERSS). The estimators and test cannot be written in closed form. Therefore, a modification of the maximum likelihood estimator using the technique suggested by Maharota and Nanda (1974) was considered. It was used to modify the likelihood ratio test to get a test in closed form for testing a simple hypothesis against one-sided alternatives.

Al-Omari and Al-Hadhrami (2011) used ERSS to estimate the parameters and population mean of the modified Weibull distribution. The maximum likelihood estimators are investigated and compared to the corresponding one based on SRS. It was found that the estimators based on ERSS are more efficient than estimators using SRS. The ERSS estimator of the population mean was also found to be more efficient than the SRS based on the same number of measured units.

Haq et al. (2013) proposed a partial ranked set sampling (PRSS) method for estimation of population mean, median and variance. On the basis of perfect and imperfect rankings, Monte Carlo simulations from symmetric and asymmetric distributions are used to evaluate the effectiveness of the proposed estimators. It was found that the estimators under PRSS are more efficient than the estimators based on simple random sampling.

Abu-Dayyeh et al. (2013) used RSS for studying the estimation of the shape and location parameters of the Pareto distribution. The estimators were compared with their counterpart in SRS in terms of their biases and mean square errors. It was shown that the estimators based on RSS can be real competitors against those based on SRS.

Sarikavanij et al. (2014) considered simultaneous comparison of the location and scale estimators of a two-parameter exponential distribution based on SRS and RSS by using generalized variance (GV). They suggested various RSS strategies to estimate the scale parameter. Their performances in terms of GV were compared with SRS strategy. It was shown that the minimum values of set size, $m$, based on RSS, which would result in smaller GV than that based on SRS. 


\section{ESTIMATION FOR PARAMETERS OF THE EED USING MRSS}

\section{Maximum likelihood estimation and fisher information based on SRS}

Consider a random sample coming from the $\operatorname{EED} f(x ; \alpha ; \lambda)$ where the values of $\alpha$ and $\lambda$ are unknown. The likelihood function is given by

$$
L_{S R S}(\alpha, \lambda)=\alpha^{n} \lambda^{n} \prod_{i=1}^{n}\left(1-e^{-\lambda x_{i}}\right)^{\alpha-1} e^{-\lambda \sum_{i=1}^{n} x_{i}} ; \quad \alpha>0, \lambda>0 .
$$

Thus, the log likelihood function is

$$
\log L_{S R S}(\alpha, \lambda)=n \log \alpha+n \log \lambda+(\alpha-1) \sum_{i=1}^{n} \log \left(1-e^{-\lambda x_{i}}\right)-\lambda \sum_{i=1}^{n} x_{i}
$$

The normal equations become

$$
\begin{gathered}
\frac{\partial \log L_{S R S}(\alpha, \lambda)}{\partial \lambda}=\frac{n}{\lambda}+(\alpha-1) \sum_{i=1}^{n} \frac{x_{i} e^{-\lambda x_{i}}}{1-e^{-\lambda x_{i}}}-\sum_{i=1}^{n} x_{i}=0 \\
\frac{\partial \log L_{S R S}(\alpha, \lambda)}{\partial \alpha}=\frac{n}{\alpha}+\sum_{i=1}^{n} \log \left(1-e^{-\lambda x_{i}}\right)=0
\end{gathered}
$$

From Equation 4, the maximum likelihood estimator (MLE) of $\alpha$ as a function of $\lambda$, say $\hat{\alpha}(\lambda)$, is

$$
\hat{\alpha}(\lambda)=\frac{-n}{\sum_{i=1}^{n} \log \left(1-e^{-\lambda x_{i}}\right)}
$$

Substituting $\hat{\alpha}(\lambda)$ in Equation 2, we obtain the profile log-likelihood of $\lambda$ as

$$
\begin{aligned}
\log L_{S R S}(\hat{\alpha}(\lambda), \lambda)= & n \log n-n \log -\sum_{i=1}^{n} \log \left(1-e^{-\lambda x_{i}}\right) \\
& +n \log \lambda-n-\sum_{i=1}^{n} \log \left(1-e^{-\lambda x_{i}}\right)-\lambda \sum_{i=1}^{n} x_{i} .
\end{aligned}
$$

The MLE of $\lambda$, can be obtained by maximizing (5) w.r.t $\lambda$ as 


$$
\frac{\partial \log L_{S R S}(\hat{\alpha}(\lambda), \lambda)}{\partial \lambda}=\frac{n}{\lambda}-\sum_{i=1}^{n} \frac{x_{i} e^{-\lambda x_{i}}}{1-e^{-\lambda x_{i}}}-\sum_{i=1}^{n} x_{i}-\frac{n}{\sum_{i=1}^{n} \log \left(1-e^{-\lambda x_{i}}\right)} \sum_{i=1}^{n} \frac{x_{i} e^{-\lambda x_{i}}}{1-e^{-\lambda x_{i}}}
$$

However, the solutions are not in closed forms, in order to obtain estimates for $\alpha$ and $\lambda$, the normal equations can be solved numerically.

Fisher information (FI) number is used to measure the amount of information that an observable sample carries about the parameter(s). The $F I$ number for the parameter $\theta$ is defined as

$$
F I(\theta)=\frac{\partial^{2} \log L(\theta)}{\partial \theta^{2}}
$$

Based on the random sample $X_{1}, X_{2}, \ldots, X_{n}$ the $F I$ numbers of $\alpha$ and $\lambda$ are, respectively, given by

$$
\begin{aligned}
& F I_{S R S}(\alpha)=\frac{\partial^{2} \log L_{S R S}(\alpha, \lambda)}{\partial \alpha^{2}}=\frac{n}{\alpha^{2}} \\
& F I_{S R S}(\lambda)=\frac{\partial^{2} \log L_{S R S}(\alpha, \lambda)}{\partial \lambda^{2}}=\frac{n}{\lambda^{2}}+(\alpha-1) \sum_{i=1}^{n} \frac{x_{i}^{2} e^{-\lambda x_{i}}}{\left(1-e^{-\lambda x_{i}}\right)^{2}}
\end{aligned}
$$

\section{Maximum likelihood estimation and fisher information based on MRSS}

Consider the maximum likelihood estimation of the parameters $\alpha$ and $\lambda$ of EED under MRSS paying attention to the odd and even set sizes.

\section{Odd set sizes}

Suppose $\left\{Y_{j i} ; j=1,2, \ldots, m\right\}$ is a MRSS from an EED, where $h$ is the number of cycles and $m$ is the set size. Since the set size $m$ is assumed to be odd, the $Y_{j i}$ are independent and identically distributed as the distribution of the $\left(\frac{m+1}{2}\right)^{\text {th }}$ order statistics of the random sample $X_{1}, X_{2}, \ldots, X_{m}$; that is 


\section{ESTIMATION FOR PARAMETERS OF THE EED USING MRSS}

$$
f_{Y}(y)=f_{X_{\left(\frac{m+1}{2}\right)}}(y)=\frac{m !}{\left(\left(\frac{m-1}{2}\right) !\right)^{2}} \alpha \lambda e^{-\lambda y}\left(1-e^{-\lambda y}\right)^{\alpha\left(\frac{m+1}{2}\right)-1}\left(1-\left(1-e^{-\lambda y}\right)^{\alpha}\right)^{\frac{m-1}{2}} .
$$

The likelihood function of MRSS for odd set size $m$ is given by

$$
\begin{aligned}
L_{\text {MRSSO }}(\alpha, \lambda)= & \prod_{j=1}^{h} \prod_{i=1}^{m} f_{Y}\left(y_{j i}\right)=c_{1} \alpha^{m h} \lambda^{m h} e^{-\lambda \sum_{j=1}^{h} \sum_{i=1}^{m} y_{j i}} \\
& \times \prod_{j=1}^{h} \prod_{i=1}^{m}\left(1-e^{-\lambda y_{j i}}\right)^{\alpha\left(\frac{m+1}{2}\right)^{-1}} \prod_{j=1}^{h} \prod_{i=1}^{m}\left(1-\left(1-e^{-\lambda y_{j i}}\right)^{\alpha}\right)^{\frac{m-1}{2}}
\end{aligned}
$$

where $c_{1}$ is a constant. Thus, the log likelihood function is

$$
\begin{aligned}
\log L_{\text {MRSSO }}(\alpha, \lambda)= & d_{1}+m h \log \alpha+m h \log \lambda-\lambda \sum_{j=1}^{h} \sum_{i=1}^{m} y_{j i} \\
& +\sum_{j=1}^{h} \sum_{i=1}^{m} y_{j i}\left(\alpha\left(\frac{m+1}{2}\right)-1\right) \sum_{j=1}^{h} \sum_{i=1}^{m}\left(1-e^{\lambda y_{j i}}\right) \\
& +\left(\frac{m-1}{2}\right) \sum_{j=1}^{h} \sum_{i=1}^{m} \log \left(1-\left(1-e^{-\lambda y_{j i}}\right)^{\alpha}\right)
\end{aligned}
$$

where $d_{1}$ is a constant.

The normal equations become

$$
\begin{aligned}
\frac{\partial \log L_{M R S S O}(\alpha, \lambda)}{\partial \alpha}= & \frac{m h}{\alpha}-\sum_{j=1}^{h} \sum_{i=1}^{m} y_{j i} \\
& +\left(\alpha\left(\frac{m+1}{2}\right)-1\right) \sum_{j=1}^{h} \sum_{i=1}^{m} \frac{y_{j i} e^{-\lambda y_{j i}}}{1-e^{\lambda y_{j i}}} \\
& -\alpha\left(\frac{m-1}{2}\right) \sum_{j=1}^{h} \sum_{i=1}^{m} \frac{y_{j i} e^{\lambda y_{j i}}\left(1-e^{\lambda y_{j i}}\right)^{\alpha-1}}{1-\left(1-e^{\lambda y_{j i}}\right) \alpha}=0
\end{aligned}
$$




$$
\begin{aligned}
\frac{\partial \log L_{M R S S O}(\alpha, \lambda)}{\partial \alpha} & =\frac{m h}{\alpha}+\left(\frac{m+1}{2}\right) \sum_{j=1}^{h} \sum_{i=1} \log \left(1-e^{-\lambda y_{j i}}\right) \\
& -\left(\frac{m-1}{2}\right) \sum_{j=1}^{h} \sum_{i=1} \frac{\left(1-e^{-\lambda y_{j i}}\right)^{\alpha} \log \left(1-e^{-\lambda y_{j i}}\right)}{1-\left(1-e^{-\lambda y_{j i}}\right)^{\alpha}}=0
\end{aligned}
$$

The MLEs of the parameters $\alpha$ and $\lambda$ are the solutions of the Equations (9) and (10). However, the solutions are not in closed forms, in order to obtain estimates for $\alpha$ and $\lambda$, the normal equations can be solved numerically. Based on the MRSS $\left\{Y_{j i} ; j=1,2, \ldots, h ; i=1,2, \ldots, m\right\}$, for odd set size $m$, the $F I$ numbers of $\alpha$ and $\lambda$ are, respectively, given by

$$
\begin{aligned}
& F I_{\text {MRSSO }}(\alpha)=\frac{\partial^{2} \log L_{\text {MRSSO }}(\alpha, \lambda)}{\partial \alpha^{2}}, \\
& F I_{\text {MRSSO }}(\lambda)=\frac{\partial^{2} \log L_{M R S S O}(\alpha, \lambda)}{\partial \lambda^{2}} .
\end{aligned}
$$

The observed FI numbers are evaluated at the maximum likelihood estimates.

\section{Even set sizes}

Because the set size $m$ is assumed to be even, for each $j=1,2, \ldots, h ; Y_{j i} \sim f_{Y_{i}}(y)$,

$$
f_{Y_{i}}(y)= \begin{cases}f_{X_{\left(\frac{m}{2}\right)}}(y) & \text { for } i=1, \ldots, \frac{m}{2} \\ f_{X_{\left(\frac{m+2}{2}\right)}}(y) & \text { for } i=\frac{m+2}{2}, \ldots, m\end{cases}
$$

where $X_{\left(\frac{m}{2}\right)}$ and $X_{\left(\frac{m+2}{2}\right)}$ are the $\left(\frac{m}{2}\right)^{\text {th }}$ and the $\left(\frac{m+2}{2}\right)^{\text {th }}$ order statistics of the random sample $X_{1}, X_{2}, \ldots, X_{m}$; therefore, for $i=1, \ldots, \frac{m}{2} ; Y_{j i}$ are independent and identically distributed as 


$$
\begin{gathered}
f_{Y_{i}}(y)=\frac{m !}{\frac{m}{2}\left(\left(\frac{m}{2}-1\right) !\right)^{2}} \alpha \lambda e^{-\lambda y}\left(1-e^{-\lambda y}\right)^{\alpha \frac{m}{2}-1}\left(1-\left(1-e^{-\lambda y}\right)^{\alpha}\right)^{\frac{m}{2}}, \\
f_{Y_{i}}(y)=\frac{m !}{\frac{m}{2}\left(\left(\frac{m}{2}-1\right) !\right)^{2}} \alpha \lambda e^{-\lambda y}\left(1-e^{-\lambda y}\right)^{\alpha\left(\frac{m}{2}+1\right)-1}\left(1-\left(1-e^{-\lambda y}\right)^{\alpha}\right)^{\frac{m}{2}-1} .
\end{gathered}
$$

and for $i=\frac{m+2}{2}, \ldots, m ; Y_{j i}$ are independent and identically distributed as

Note $\left\{Y_{j i} ; j=1,2, \ldots, h ; i=1,2, \ldots, m\right\}$ are independent. Thus, the likelihood function of MRSS for even set size $m$ is given by

$$
\begin{aligned}
L_{M R S S E}(\alpha, \lambda)= & \left(\prod_{j=1}^{h} \prod_{i=1}^{\frac{m}{2}} f_{Y_{i}}\left(y_{j i}\right) \times\left(\prod_{j=1}^{h} \prod_{i=\frac{m+2}{2}}^{m} f_{Y_{i}}\left(y_{j i}\right)\right)\right. \\
& =c_{2} \alpha^{m h} \lambda^{m h} e^{-\lambda \sum_{j=1}^{h} \sum_{i=1}^{\frac{m}{2}} y_{j i}} \times e^{-\lambda \sum_{j=1}^{h} \sum_{i=\frac{m+2}{2}}^{\frac{m}{2}} y_{j i}} \prod_{j=1}^{h} \prod_{i=1}^{\frac{m}{2}}\left(1-e^{-\lambda y_{j i}}\right)^{\alpha \frac{m}{2}-1} \\
& \times \prod_{j=1}^{h} \prod_{i=\frac{m+2}{2}}^{m}\left(1-e^{-\lambda y_{j i}}\right)^{\alpha\left(\frac{m}{2}+1\right)-1} \prod_{j=1}^{h} \prod_{i=1}^{\frac{m}{2}}\left(1-\left(1-e^{-\lambda y_{j i}}\right)^{\alpha}\right)^{\frac{m}{2}} \\
& \prod_{j=1}^{h} \prod_{i=\frac{m+2}{2}}^{m}\left(1-\left(1-e^{-\lambda y_{j i}}\right)^{\alpha}\right)^{\frac{m}{2}-1},
\end{aligned}
$$

where $c_{2}$ is a constant. Thus, the log likelihood function is 


\section{SAMUH \& QTAIT}

$\log L_{M R S S E}(\alpha, \lambda)=d_{2}+m h \log \alpha+m h \log \lambda$

$$
\begin{aligned}
& -\lambda \sum_{j=1}^{h} \sum_{i=1}^{\frac{m}{2}} y_{j i}-\lambda \sum_{j=1}^{h} \sum_{i=\frac{m+2}{2}}^{m} y_{j i} \\
& +\left(\alpha \frac{m}{2}-1\right) \sum_{j=1}^{h} \sum_{i=1}^{\frac{m}{2}} \log \left(1-e^{-\lambda y_{j i}}\right) \\
& +\left(\alpha\left(\frac{m}{2}+1\right)-1\right) \sum_{j=1}^{h} \sum_{i=\frac{m+2}{2}} \log \left(1-e^{-\lambda y_{j i}}\right) \\
& +\frac{m}{2} \sum_{j=1}^{h} \sum_{i=1}^{\frac{m}{2}} \log \left(1-\left(1-e^{-\lambda y_{j i}}\right)^{\alpha}\right) \\
& +\left(\frac{m}{2}-1\right) \sum_{j=1}^{h} \sum_{i=\frac{m+2}{2}}^{m} \log \left(1-\left(1-e^{-\lambda y_{j i}}\right)^{\alpha}\right),
\end{aligned}
$$

where $d_{2}$ is a constant.

The normal equations become

$$
\begin{aligned}
\log L_{\text {MRSSE }}(\alpha, \lambda)= & d_{2}+m h \log \alpha+m h \log \lambda \\
& -\lambda \sum_{j=1}^{h} \sum_{i=1}^{\frac{m}{2}} y_{j i}-\lambda \sum_{j=1}^{h} \sum_{i=\frac{m+2}{2}}^{m} y_{j i} \\
& +\left(\alpha \frac{m}{2}-1\right) \sum_{j=1}^{h} \sum_{i=1}^{\frac{m}{2}} \log \left(1-e^{-\lambda y_{j i}}\right) \\
& +\left(\alpha\left(\frac{m}{2}+1\right)-1\right) \sum_{j=1}^{h} \sum_{i=\frac{m+2}{2}} \log \left(1-e^{-\lambda y_{j i}}\right) \\
& +\frac{m}{2} \sum_{j=1}^{h} \sum_{i=1}^{\frac{m}{2}} \log \left(1-\left(1-e^{-\lambda y_{j i}}\right)^{\alpha}\right) \\
& +\left(\frac{m}{2}-1\right) \sum_{j=1}^{h} \sum_{i=\frac{m+2}{2}}^{m} \log \left(1-\left(1-e^{-\lambda y_{j i}}\right)^{\alpha}\right),
\end{aligned}
$$




\section{ESTIMATION FOR PARAMETERS OF THE EED USING MRSS}

$$
\begin{aligned}
\frac{\partial \log L_{M R S S E}(\alpha, \lambda)}{\partial \alpha}= & \frac{m h}{\alpha}+\frac{m}{2} \sum_{j=1}^{h} \sum_{i=1}^{\frac{m}{2}} \log \left(1-e^{-\lambda y_{j i}}\right) \\
& +\left(\frac{m}{2}+1\right) \sum_{j=1}^{h} \sum_{i=\frac{m+2}{2}} \log \left(1-e^{-\lambda y_{j i}}\right) \\
& -\frac{m}{2} \sum_{j=1}^{h} \sum_{i=1}^{\frac{m}{2}} \frac{\left(1-e^{-\lambda y_{j i}}\right)^{\alpha} \log \left(1-e^{-\lambda y_{j i}}\right)}{1-\left(1-e^{-\lambda y_{j i}}\right)^{\alpha}} \\
& -\left(\frac{m}{2}-1\right) \sum_{j=1}^{h} \sum_{i=\frac{m+2}{2}}^{\frac{m}{2}} \frac{\left(1-e^{-\lambda y_{j i}}\right)^{\alpha} \log \left(1-e^{-\lambda y_{j i}}\right)}{1-\left(1-e^{-\lambda y_{j i}}\right)^{\alpha}} \\
& =0
\end{aligned}
$$

The MLEs of the parameters $\alpha$ and $\lambda$ are the solutions of the Equations (15) and (16). However, the solutions are not in closed forms, in order to obtain estimates for $\alpha$ and $\lambda$, the normal equations can be solved numerically. Based on the $\operatorname{MRSS}\left\{Y_{j i} ; j=1,2, \ldots, h ; i=1,2, \ldots, m\right\}$, for even set size $m$, the $F I$ numbers of $\alpha$ and $\lambda$ are, respectively, given by

$$
\begin{aligned}
& F I_{\text {MRSSE }}(\alpha)=\frac{\partial^{2} \log L_{\text {MRSSE }}(\alpha, \lambda)}{\partial \alpha^{2}}, \\
& F I_{\text {MRSSE }}(\lambda)=\frac{\partial^{2} \log L_{M R S S E}(\alpha, \lambda)}{\partial \lambda^{2}} .
\end{aligned}
$$

The observed FI numbers are evaluated at the maximum likelihood estimates.

The comparison between the resulting estimators under MRSS and SRS can be done using the asymptotic efficiency (see Stokes, 1995). The asymptotic efficiency of MRSS w.r.t SRS for estimating $\theta$ is defined by

$$
\operatorname{Aeff}\left(\hat{\theta}_{M R S S} ; \hat{\theta}_{S R S}\right)=\lim _{n \rightarrow \infty} \operatorname{eff}\left(\hat{\theta}_{M R S S} ; \hat{\theta}_{S R S}\right)=\frac{F I_{M R S S}(\theta)}{F I_{S R S}(\theta)}
$$




\section{SAMUH \& QTAIT}

\section{Interval Estimates}

Let $X_{1}, \ldots, X_{n}$ be a random sample from $f(x ; \theta)$, where $\theta$ is an unknown quantity. A confidence interval for the parameter $\theta$, with confidence level or confidence coefficient $1-\gamma, \quad$ is an interval with random endpoints $\left[S_{L}\left(X_{1}, \ldots, X_{n}\right), S_{u}\left(X_{1}, \ldots, X_{n}\right)\right]$. It is given by

$$
P\left(S_{L}\left(X_{1}, \ldots, X_{n}\right) \leq \theta \leq S_{U}\left(X_{1}, \ldots, X_{n}\right)\right)=1-\gamma
$$

The interval $\left[S_{L}\left(X_{1}, \ldots, X_{n}\right), S_{u}\left(X_{1}, \ldots, X_{n}\right)\right]$ is called a $100(1-\gamma) \%$ confidence interval for $\theta$.

For large sample size, the maximum likelihood estimator, under appropriate regularity conditions (see Davison, 2008, p.118), has many useful properties, including reparametrization-invariance, consistency, efficiency, and the sampling

distribution of a maximum likelihood estimator $\hat{\theta}_{M L E}$ is asymptotically unbiased and also asymptotically normal with its variance obtained from the inverse Fisher information number of sample size 1 at the unknown parameter $\theta$; that is, $\hat{\theta}_{M L E} \rightarrow N\left(\theta, F I^{-1}(\theta)\right)$ as $n \rightarrow \infty$. Therefore, the approximate $100(1-\gamma) \%$ confidence limits for the $\hat{\theta}_{M L E}$ of $\theta$ can be constructed as

$$
P\left(-z_{\frac{\gamma}{2}} \leq \frac{\hat{\theta}-\theta}{\sqrt{F I^{-1}(\theta)}} \leq z_{\frac{\gamma}{2}}\right)=1-\gamma
$$

where $z_{\gamma}$ is the $\gamma^{\text {th }}$ upper percentile of the standard normal distribution. Therefore, the approximate $100(1-\gamma) \%$ confidence limits for the scale and location parameters of the EED are given, respectively, by

$$
\begin{aligned}
& P\left(\hat{\alpha}-z_{\frac{\gamma}{2}} \sqrt{F I^{-1}(\alpha)} \leq \alpha \leq \hat{\alpha}+z_{\frac{\gamma}{2}} \sqrt{F I^{-1}(\alpha)}\right)=1-\gamma, \\
& P\left(\hat{\lambda}-z_{\frac{\gamma}{2}} \sqrt{F I^{-1}(\lambda)} \leq \lambda \leq \hat{\lambda}+z_{\frac{\gamma}{2}} \sqrt{F I^{-1}(\lambda)}\right)=1-\gamma .
\end{aligned}
$$

Then, the approximate confidence limits for $\alpha$ and $\lambda$ will be constructed using Equation (17) and (18), respectively. 


\section{ESTIMATION FOR PARAMETERS OF THE EED USING MRSS}

\section{Simulation Study}

To investigate the properties of the maximum likelihood estimators of the scale and locations parameters of the EED a simulation study is conducted. Monte Carlo simulation is applied for different sample sizes, $m=\{2,3,4,5\}$ and $h=\{10,50,100\}$, and for different parameter values, $(\alpha, \lambda)=\{(1,1),(0.5,1.5),(1.5,2.5)\}$. The estimates of $\alpha$ and $\lambda$, the bias estimates, the MSEs, and the efficiency values are computed over 2000 replications for different cases. The results are reported in Tables 2-4. Moreover, the observed Fisher information matrices and the asymptotic efficiency in estimating $\alpha$ and $\lambda$ under SRS and MRSS are calculated and the results reported in Table 5. The observed Fisher information numbers of $\alpha$ and $\lambda$ based on SRS are denoted by $F_{S R S}(\hat{\alpha})$ and $F I_{S R S}(\hat{\lambda})$, respectively, and the observed information numbers of $\alpha$ and $\lambda$ based on MRSS are denoted by $F I_{\text {MRSS }}(\hat{\alpha})$ and $F I_{M R S S}(\hat{\lambda})$, respectively. The asymptotic efficiency, Aeff, for estimating $\alpha$ is found as the ratio

$$
\operatorname{Aeff}\left(\hat{\alpha}_{M R S S} ; \hat{\alpha}_{S R S}\right)=\frac{F I_{M R S S}(\alpha)}{F I_{S R S}(\alpha)},
$$

and for estimating $\lambda$ is found as the ratio

$$
\operatorname{Aeff}\left(\hat{\lambda}_{M R S S} ; \hat{\lambda}_{S R S}\right)=\frac{F I_{M R S S}(\lambda)}{F I_{S R S}(\lambda)}
$$

Confidence intervals based on SRS and MRSS for $(\alpha, \lambda)=(1.5,2.5)$ for different sample sizes are constructed at $1-\gamma=0.95$ level of confidence using Equation (17) and (18), respectively, and the results are shown in Table 5. 


\section{SAMUH \& QTAIT}

Table 2. The Bias, MSE, and Efficiency values of estimating the parameters $(\alpha=1, \lambda=1)$ under SRS and MRSS.

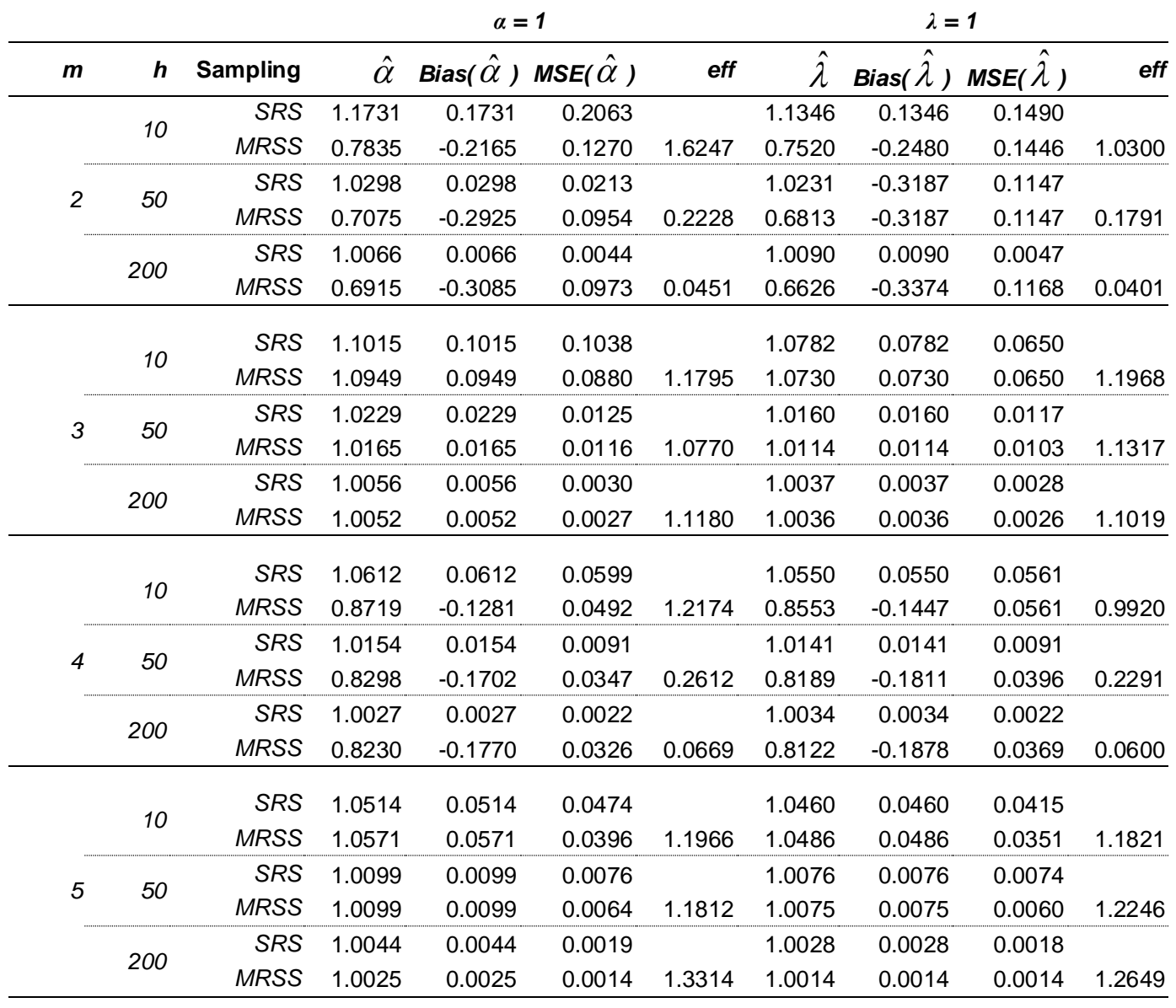




\section{ESTIMATION FOR PARAMETERS OF THE EED USING MRSS}

Table 3. The Bias, MSE, and Efficiency values of estimating the parameters $(\alpha=0.5$, $\lambda=1.5)$ under SRS and MRSS.

\begin{tabular}{|c|c|c|c|c|c|c|c|c|c|c|}
\hline & & & \multicolumn{4}{|c|}{$\alpha=0.5$} & \multicolumn{4}{|c|}{$\lambda=1.5$} \\
\hline$m$ & $\boldsymbol{h}$ & Sampling & $\hat{\alpha}$ & $\operatorname{Bias}(\hat{\alpha})$ & $\operatorname{MSE}(\hat{\alpha})$ & eff & $\hat{\lambda}$ & $\operatorname{Bias}(\hat{\lambda})$ & $\operatorname{MSE}(\hat{\lambda})$ & ef \\
\hline \multirow{6}{*}{2} & \multirow{2}{*}{10} & SRS & 0.5687 & 0.0687 & 0.0349 & & 1.7938 & 0.2938 & 0.6328 & \\
\hline & & MRSS & 0.4062 & -0.0938 & 0.0240 & 1.4557 & 1.0823 & -0.4177 & 0.4572 & 1.3842 \\
\hline & \multirow{2}{*}{50} & SRS & 0.5124 & 0.0124 & 0.0041 & & 1.5499 & 0.0499 & 0.0730 & \\
\hline & & MRSS & 0.3748 & -0.1252 & 0.0178 & 0.2317 & 0.9367 & -0.5633 & 0.3566 & 0.2048 \\
\hline & \multirow{2}{*}{200} & SRS & 0.5024 & 0.0024 & 0.0009 & & 1.5181 & 0.0181 & 0.0160 & \\
\hline & & MRSS & 0.3680 & -0.1320 & 0.0179 & 0.0493 & 0.9006 & -0.5994 & 0.3676 & 0.0445 \\
\hline \multirow{6}{*}{3} & \multirow{2}{*}{10} & SRS & 0.5410 & 0.0410 & 0.0188 & & 1.6688 & 0.1688 & 0.3043 & \\
\hline & & MRSS & 0.5366 & 0.0366 & 0.0146 & 1.2872 & 1.6565 & 0.1565 & 0.2594 & 1.1732 \\
\hline & \multirow{2}{*}{50} & SRS & 0.5100 & 0.0100 & 0.0025 & & 1.5341 & 0.0346 & 0.0418 & \\
\hline & & MRSS & 0.5067 & 0.0067 & 0.0021 & 1.1871 & 1.5246 & 0.0246 & 0.0382 & 1.0930 \\
\hline & \multirow{2}{*}{200} & SRS & 0.5024 & 0.0024 & 0.0006 & & 1.5080 & 0.0080 & 0.0099 & \\
\hline & & MRSS & 0.5021 & 0.0021 & 0.0005 & 1.2382 & 1.5077 & 0.0077 & 0.0094 & 1.0532 \\
\hline \multirow{6}{*}{4} & \multirow{2}{*}{10} & SRS & 0.5238 & 0.0238 & 0.0110 & & 1.6165 & 0.1165 & 0.2066 & \\
\hline & & MRSS & 0.4463 & -0.0537 & 0.0090 & 1.2301 & 1.2424 & -0.2576 & 0.1895 & 1.0905 \\
\hline & \multirow{2}{*}{50} & SRS & 0.5062 & 0.0062 & 0.0018 & & 1.5284 & 0.0284 & 0.0318 & \\
\hline & & MRSS & 0.4290 & -0.0710 & 0.0062 & 0.2929 & 1.1656 & -0.3344 & 0.1345 & 0.2365 \\
\hline & \multirow{2}{*}{200} & SRS & 0.5010 & 0.0010 & 0.0004 & & 1.5070 & 0.0070 & 0.0077 & \\
\hline & & MRSS & 0.4263 & -0.0737 & 0.0057 & 0.0775 & 1.1520 & -0.3480 & 0.1263 & 0.0608 \\
\hline \multirow{6}{*}{5} & \multirow{2}{*}{10} & SRS & 0.5205 & 0.0205 & 0.0091 & & 1.5984 & 0.0984 & 0.1544 & \\
\hline & & MRSS & 0.5219 & 0.0219 & 0.0065 & 1.3956 & 1.6029 & 0.1029 & 0.1371 & 1.1265 \\
\hline & \multirow{2}{*}{50} & SRS & 0.5041 & 0.0041 & 0.0015 & & 1.5166 & 0.0166 & 0.0260 & \\
\hline & & MRSS & 0.5039 & 0.0039 & 0.0011 & 1.3306 & 1.5160 & 0.0160 & 0.0225 & 1.1578 \\
\hline & \multirow{2}{*}{200} & SRS & 0.5019 & 0.0019 & 0.0004 & & 1.5055 & 0.0055 & 0.0062 & \\
\hline & & MRSS & 0.5010 & 0.0010 & 0.0003 & 1.5124 & 1.5033 & 0.0033 & 0.0053 & 1.1736 \\
\hline
\end{tabular}




\section{SAMUH \& QTAIT}

Table 4. The Bias, MSE, and Efficiency values of estimating the parameters $(\alpha=1.5$, $\lambda=2.5)$ under SRS and MRSS.

\begin{tabular}{|c|c|c|c|c|c|c|c|c|c|c|}
\hline \multirow[b]{2}{*}{$m$} & & & \multicolumn{4}{|c|}{$\alpha=1.5$} & \multicolumn{4}{|c|}{$\lambda=2.5$} \\
\hline & $h$ & Sampling & $\hat{\alpha}$ & $\operatorname{Bias}(\hat{\alpha})$ & $\operatorname{MSE}(\hat{\alpha})$ & eff & $\hat{\lambda}$ & $\operatorname{Bias}(\hat{\lambda})$ & $\operatorname{MSE}(\hat{\lambda})$ & eff \\
\hline \multirow{6}{*}{2} & \multirow{2}{*}{10} & SRS & 1.8041 & 0.3041 & 0.6164 & & 2.7886 & 0.2886 & 0.7157 & \multirow[b]{2}{*}{0.9304} \\
\hline & & MRSS & 1.1505 & -0.3495 & 0.3413 & 1.8061 & 1.9210 & -0.5790 & 0.7692 & \\
\hline & \multirow{2}{*}{50} & SRS & 1.5507 & 0.0507 & 0.0575 & & 2.5493 & 0.0493 & 0.1040 & \multirow[b]{2}{*}{0.1710} \\
\hline & & MRSS & 1.0212 & -0.4788 & 0.2538 & 0.2264 & 1.7673 & -0.7327 & 0.6082 & \\
\hline & \multirow{2}{*}{200} & SRS & 1.5119 & 0.0119 & 0.0116 & & 2.5198 & 0.0198 & 0.0238 & \multirow[b]{2}{*}{0.0387} \\
\hline & & MRSS & 0.9948 & -0.5052 & 0.2605 & 0.0445 & 1.7256 & -0.7744 & 0.6157 & \\
\hline \multirow{6}{*}{3} & \multirow{2}{*}{10} & SRS & 1.6762 & 0.1762 & 0.2947 & & 2.6681 & 0.1681 & 0.3835 & \multirow[b]{2}{*}{1.1976} \\
\hline & & MRSS & 1.6680 & 0.1680 & 0.2600 & 1.1336 & 2.6581 & 0.1581 & 0.3202 & \\
\hline & \multirow{2}{*}{50} & SRS & 1.5380 & 0.0380 & 0.0329 & & 2.5341 & 0.0341 & 0.0589 & \multirow[b]{2}{*}{1.1341} \\
\hline & & MRSS & 1.5285 & 0.0285 & 0.0320 & 1.0270 & 2.5245 & 0.0245 & 0.0520 & \\
\hline & \multirow{2}{*}{200} & SRS & 1.5092 & 0.0092 & 0.0078 & & 2.5080 & 0.0080 & 0.0145 & \multirow[b]{2}{*}{1.1103} \\
\hline & & MRSS & 1.5088 & 0.0088 & 0.0074 & 1.0628 & 2.5078 & 0.0078 & 0.0130 & \\
\hline \multirow{6}{*}{4} & \multirow{2}{*}{10} & SRS & 1.6086 & 0.1086 & 0.1688 & & 2.6194 & 0.1194 & 0.2785 & \multirow[b]{2}{*}{0.9568} \\
\hline & & MRSS & 1.2888 & -0.2112 & 0.1343 & 1.2568 & 2.1679 & -0.3321 & 0.2911 & \\
\hline & \multirow{2}{*}{50} & SRS & 1.5267 & 0.0267 & 0.0241 & & 2.5311 & 0.0311 & 0.0461 & \multirow[b]{2}{*}{0.2255} \\
\hline & & MRSS & 1.2173 & -0.2827 & 0.0951 & 0.2531 & 2.0889 & -0.4111 & 0.2045 & \\
\hline & \multirow{2}{*}{200} & SRS & 1.5049 & 0.0049 & 0.0057 & & 2.5074 & 0.0074 & 0.0113 & \multirow[b]{2}{*}{0.0594} \\
\hline & & MRSS & 1.2054 & -0.2946 & 0.0901 & 0.0636 & 2.0742 & -0.4258 & 0.1897 & \\
\hline \multirow{6}{*}{5} & \multirow{2}{*}{10} & SRS & 1.5897 & 0.0897 & 0.1286 & & 2.5990 & 0.0990 & 0.2071 & \multirow[b]{2}{*}{1.1900} \\
\hline & & MRSS & 1.6012 & 0.1012 & 0.1160 & 1.1095 & 2.6059 & 0.1059 & 0.1740 & \\
\hline & \multirow{2}{*}{50} & SRS & 1.5169 & 0.0169 & 0.0201 & & 2.5163 & 0.0163 & 0.0375 & \multirow[b]{2}{*}{1.2210} \\
\hline & & MRSS & 1.5172 & 0.0172 & 0.0181 & 1.1081 & 2.5160 & 0.0160 & 0.0307 & \\
\hline & \multirow{2}{*}{200} & SRS & 1.5073 & 0.0073 & 0.0050 & & 2.5063 & 0.0063 & 0.0093 & \\
\hline & & MRSS & 1.5041 & 0.0041 & 0.0040 & 1.2501 & 2.5030 & 0.0030 & 0.0072 & 1.2845 \\
\hline
\end{tabular}




\section{ESTIMATION FOR PARAMETERS OF THE EED USING MRSS}

Table 5. The observed Fisher information matrix, the variance-covariance matrix, a $95 \%$ confidence interval of the parameters $(\alpha=1.5, \lambda=2.5)$, and the asymptotic efficiency under SRS and MRSS.

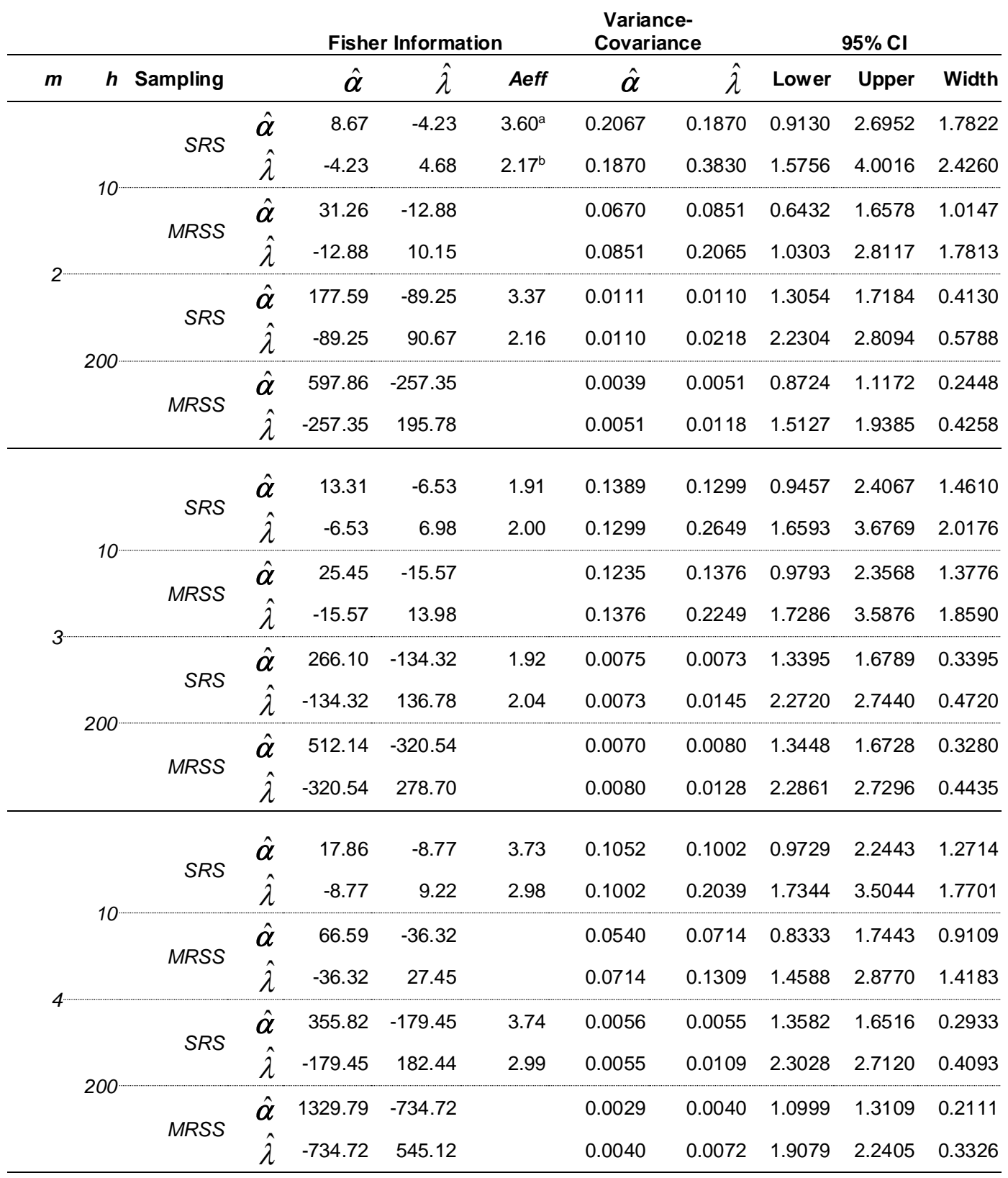

(continued) 
SAMUH \& QTAIT

\begin{tabular}{|c|c|c|c|c|c|c|c|c|c|c|}
\hline \multirow{4}{*}{10} & \multirow{2}{*}{ SRS } & $\hat{\alpha}$ & 22.49 & -11.06 & 2.79 & 0.0843 & 0.0811 & 1.0206 & 2.1588 & 1.1382 \\
\hline & & $\hat{\lambda}$ & -11.06 & 11.49 & 3.06 & 0.0811 & 0.1651 & 1.8026 & 3.3954 & 1.5928 \\
\hline & \multirow{2}{*}{ MRSS } & $\hat{\alpha}$ & 62.70 & -41.62 & & 0.0748 & 0.0886 & 1.0651 & 2.1373 & 1.0721 \\
\hline & & $\hat{\lambda}$ & -41.62 & 35.12 & & 0.0886 & 0.1335 & 1.8898 & 3.3220 & 1.4323 \\
\hline \multirow{4}{*}{200} & \multirow{2}{*}{ SRS } & $\hat{\alpha}$ & 443.02 & -223.87 & 2.88 & 0.0045 & 0.0044 & 1.3758 & 1.6388 & 0.2630 \\
\hline & & $\hat{\lambda}$ & -223.87 & 227.76 & 3.11 & 0.0044 & 0.0087 & 2.3235 & 2.6891 & 0.3656 \\
\hline & \multirow{2}{*}{ MRSS } & $\hat{\alpha}$ & 1277.40 & -854.20 & & 0.0041 & 0.0049 & 1.3786 & 1.6296 & 0.2510 \\
\hline & & $\hat{\lambda}$ & -854.20 & 707.46 & & 0.0049 & 0.0073 & 2.3355 & 2.6705 & 0.3349 \\
\hline
\end{tabular}

${ }^{*}$ Note: a) Aeff ( $\left.\hat{\boldsymbol{\alpha}}_{\text {MRSS }}, \hat{\boldsymbol{\alpha}}_{S R S}\right)$, b) Aeff $\left(\hat{\lambda}_{\text {MRSS }}, \hat{\lambda}_{S R S}\right)$

\section{Conclusion}

The method of maximum likelihood estimation for estimating the shape and scale parameters of the EED is studied in the MRSS framework. The new obtained estimators are com-pared with the conventional estimators obtained by SRS. The relative efficiency are calculated for comparing the estimators. The amount of information available from the MRSS about the parameters of the EED is evaluated. Confidence intervals for the parameters are constructed using SRS and MRSS. More specifically, we have the following conclusions.

1. From Tables 2-4 it can be concluded that:

a. For each sampling method, the MSEs of the estimators decrease as the set size increases and as the number of cycle increases.

b. It is clear, from the biases, that MRSS overestimate and when the set size is odd and underestimate and when the set size is even.

c. The biases of the estimators based on MRSS when the set size is odd decrease as the number of cycle increases. When the set size is even the biases of the estimators based on MRSS increase as the number of cycle increases.

d. The efficiency is always greater than 1 when the set size is odd; that is, MRSS is more efficient than SRS in estimating the parameters of the EED.

2. From Table 5 it can be concluded that: 


\section{ESTIMATION FOR PARAMETERS OF THE EED USING MRSS}

a. Fisher information numbers obtained from MRSS are greater than that from SRS.

b. The asymptotic variances of the estimators decrease as the set size increases and as the number of cycle increases.

c. The interval width of the estimators decreases as the set size increases and as the number of cycle increases.

d. The interval width obtained by MRSS is narrower than the one obtained by SRS.

\section{References}

Abu-Dayyeh, W., Assrhani, A., \& Ibrahim, K. (2013). Estimation of the shape and scale parameters of Pareto distribution using ranked set sampling. Statistical Papers, 54(1), 207-225. doi:10.1007/s00362-011-0420-3

Abu-Dayyeh, W. \& Sawi, E. A. (2009). Modified inference about the mean of the exponential distribution using moving extreme ranked set sampling. Statistical Papers, 50(2), 249-259. doi:10.1007/s00362-007-0072-5

Al-Omari, A. \& Al-Hadhrami, S. A. (2011). On maximum likelihood estimators of the parameters of a modified Weibull distribution using extreme ranked set sampling. Journal of Modern Applied Statistical Methods, 10(2), 607-617. http://digitalcommons.wayne.edu/jmasm/vol10/iss2/18/

Davison, A. C. (2008). Statistical Models. New York: Cambridge University Press.

Ghitany, M. E., Al-Jarallah, R. A., \& Balakrishnan, N. (2013). On the existence and uniqueness of the MLEs of the parameters of a general class of exponentiated distributions. Statistics: A Journal of Theoretical and Applied Statistics, 47(3), 605-612. doi:10.1080/02331888.2011.614950

Gupta, R. D. \& Kundu, D. (1999). Generalized exponential distributions. Australian and New Zealand Journal of Statistics, 41(2), 173-188. doi:10.1111/1467-842X.00072

Gupta, R. D. \& Kundu, D. (2001). Exponentiated exponential family: An alternative to gamma and Weibull distributions. Biometrical Journal, 43(1), 117-130. doi:10.1002/1521-4036(200102)43:1<117::AID-BIMJ117>3.0.CO;2-R

Haq, A., Brown, J., Moltchanova, E., \& Al-Omari, A. I. (2013). Partial ranked set sampling design. Environmetrics, 24(3), 201-207. doi:10.1002/env.2203 


\section{SAMUH \& QTAIT}

Sinha, B. K., Sinha, B. K., \& Purkayastha, S. (1996). On some aspects of ranked set sampling for estimation of normal and exponential parameters. Statistics \& Risk Modeling, 14(3), 223-240. doi:10.1524/strm.1996.14.3.223

Lam, K., Sinha, B. K., \& Wu, Z. (1994). Estimation of parameters in twoparameter exponential distribution using ranked set sampling. Annals of the Institute of Statistics and Mathematics, 46(4), 723-736. doi:10.1007/BF00773478

Maharota, K. \& Nanda, P. (1974). Unbiased estimator of parameter by order statistics in the case of censored samples. Biometrika, 61(3), 601-606. doi:10.1093/biomet/61.3.601

McIntyre, G. (1952). A method for unbiased selective sampling, using ranked sets. Australian Journal of Agricultural Research, 3(4), 385-390. doi:10.1071/AR9520385

Muttlak, H. A. (1997). Median ranked set sampling. Journal of Applied Statistical Science, 6(4), 245-255.

Nadarajah, S. (2011). The exponentiated exponential distribution: a survey. Advances in Statistical Analysis, 95(3), 219-251. doi:10.1007/s10182-011-0154-5

Ristić, M. M. \& Nadarajah, S. (2014). A new lifetime distribution. Journal of Statistical Computation and Simulation, 84(1), 135-150. doi:10.1080/00949655.2012.697163

Samawi, H. M. \& Al-Sagheer, O. (2001). On the estimation of the distribution function using extreme and median ranked set sampling. Biometrical Journal, 43(3), 357-373. doi:10.1002/1521-4036(200106)43:3<357::AID-BIMJ357>3.0.CO;2-Q

Sarikavanij, S., Kasala, S., \& Sinha, B. K. (2014). Estimation of location and scale parameters in two-parameter exponential distribution based on ranked set sample. Communications in Statistics - Simulation and Computation, 43(1), 132-141. doi:10.1080/03610918.2012.698776

Stokes, L. (1995). Parametric ranked set sampling. Annals of the Institute of Statistical Mathematics, 47(3), 465-482. doi:10.1007/BF00773396

Stokes, S. L. (1976). An investigation of the consequences of ranked set sampling (Unpublished doctoral thesis). University of North Carolina, Chapel Hill, NC. 